\title{
Jet charge in heavy-ion collisions
}

\author{
Hai Tao $\mathrm{Li}^{1, \star}$ \\ ${ }^{1}$ Theoretical Division, Los Alamos National Laboratory, Los Alamos, NM, 87545, USA
}

\begin{abstract}
.
Jet quenching effects have been widely used to study the properties of strongly-interacting matter, quark-gluon plasma, in heavy-ion collisions. Flavor tagging in heavy-ion collisions plays an important role to reveal the medium parton showers for quark and gluon evolution. Combining with kinematic information, the average jet charge can be used to separate the contribution of different jet flavors, which is defined as the momentumweighted sum of the charges of hadrons inside a given jet. Using soft-collinear effective theory with medium interactions, we investigate the factorization of the jet charge in QCD medium. We provide predictions for jet charge distributions and their modifications compared to the ones in proton-proton collisions.
\end{abstract}

\section{Introduction}

The jet charge is defined as the transverse momentum-weighted sum of the charges of the hadrons in jet cone [1]

$$
Q_{\kappa, \text { jet }}=\sum_{i \in \text { jet }} Q_{i}\left(\frac{p_{T}^{i}}{p_{T}^{\mathrm{jet}}}\right)^{\kappa},
$$

where $p_{T}^{\text {jet }}$ and $p_{T}^{i}$ is the transverse momenta of the jet and hadron $i . Q_{i}$ is the electric charge of particle $i$ and $\kappa$ is a free parameter.

In proton-proton collisions, the works [2,3] studied the jet charge distribution using soft-collinear effective theory, where this observable can be written as the product of perturbative calculable jet functions and the non-perturbative fragmentation functions. The average charge of gluon jet is approximately zero if we ignore the soft correlations. Ideally the charges of different jet flavors are different which makes it is possible to tag the flavor origin of the jet. At the LHC, the ATLAS collaboration in Ref. [4] extracted the average charges of up- and down-quark jet as a function of jet $p_{T}$, where the charges are well separated. Furthermore, they also measured the scale violation of the jet charge distribution, for which the prediction is

$$
\frac{p_{T}}{\left\langle Q_{\kappa, q}\right\rangle} \frac{d}{d p_{T}}\left\langle Q_{\kappa, q}\right\rangle=\frac{\alpha_{s}}{\pi} \tilde{P}_{q q}(\kappa),
$$

where $\tilde{P}_{q q}(\kappa)$ is the moment of the QCD splitting function.

^e-mail: haitaoli@lanl.gov 
In heavy-ion collisions, jet charge is a promising observable, which can be used to study the initial-state and final-state effects. For the initial state effects, the parton densities of up-quark and down-quark are very different in heavy nucleus resulting in different fractions of up-quark and downquark jets in comparison with proton-proton collisions. The charge distributions of untagged jets are significantly modified. For the final state effects, the gluon tends to lose more energy due to the larger color factor. Therefore, more quark jets are expected in heavy-ion collisions. Furthermore, the hot QCD medium produced in the collisions also modifies the jet and fragmentation functions. The evolution of energetic partons medium can be tested uniquely.

In these proceedings, we present our recent work [5] about jet charge in heavy-ion collisions, where we introduce a framework for perturbative calculations of the average jet charge in analogy to the study [2,3] in vacuum. The global-fit nuclear PDFs are used for the initial state effects. The final state effects are constructed by the medium-induced collinear splitting kernels [6-10]. For similar applications see Refs. [11-14]. And we will show that the initial state isospin effects and final state jet quenching effects can be disentangled utilizing jet charge distributions.

\section{Framework}

\subsection{Jet charge in proton-proton collisions}

According to the factorization of the cross section for finding a hadron inside a given jet in SCET, the average jet charge for a quark jet can be written as [2,3]

$$
\left\langle Q_{\kappa, q}\right\rangle=\frac{\tilde{\mathcal{J}}_{q q}(E, R, \kappa, \mu)}{J_{q}(E, R, \mu)} \tilde{D}_{q}^{Q}(\kappa, \mu)
$$

with $\tilde{\mathcal{J}}_{q q}(E, R, \kappa, \mu)$ the Wilson coefficient matching the fragmenting jet function onto a fragmentation function. $E, R$ and $\mu$ are the jet energy, the jet radius, and the factorization scale, respectively. The $(\kappa+1)$-th Mellin moments are defined as

$$
\tilde{\mathcal{J}}_{q q}(E, R, \kappa, \mu)=\int_{0}^{1} d z z^{\kappa} \mathcal{J}_{q q}(E, R, z, \mu), \quad \tilde{D}_{q}^{Q}(\kappa, \mu)=\int_{0}^{1} d z z^{\kappa} \sum_{h} Q_{h} D_{q}^{h}(z, \mu) .
$$

The detail discussions about the jet function and the jet matching coefficients can be found in Refs. $[3,15,16]$. For a given flavor, jet charge only depends on one nonperturbative parameter, the fragmentation function $D_{j}^{Q}$, which is the momentum weighted charge for parton $j$ evolving into all hadrons. The fragmentation function obeys a DGLAP-like evolution. Due to no contribution from gluon jet, the evolution equation of $\tilde{D}_{q}^{Q}(\kappa, \mu)$ can be written as

$$
\mu \frac{d}{d \mu} \tilde{D}_{q}^{Q}(\kappa, \mu)=\frac{\alpha_{s}(\mu)}{\pi} \tilde{P}_{q q}(\kappa) \tilde{D}_{q}^{Q}(\kappa, \mu)
$$

\subsection{Jet charge in heavy-ion collisions}

In analogy to the case of the vacuum jet function which describes the physics in the collinear limit, the medium modifications are introduced by replacing the splitting functions by the in-medium splitting ones. And the medium-induced splitting functions can be constructed using the splitting kernels 
induced by QCD medium as discussed in Refs. [5, 11, 12]. Similar to the case of semi-inclusive jet functions $[13,14]$, the medium corrections to the matching coefficients are

$$
\begin{aligned}
& \mathcal{J}_{q q}^{\mathrm{med}}(E, R, x, \mu)=\frac{\alpha_{s}(\mu)}{2 \pi^{2}} \int_{0}^{2 E x(1-x) \tan R / 2} \frac{d^{2} \mathbf{k}_{\perp}}{\mathbf{k}_{\perp}^{2}} P_{q \rightarrow q g}^{\mathrm{med}}\left(x, \mathbf{k}_{\perp}\right), \\
& \mathcal{J}_{q g}^{\mathrm{med}}(E, R, x, \mu)=\frac{\alpha_{s}(\mu)}{2 \pi^{2}} \int_{0}^{2 E x(1-x) \tan R / 2} \frac{d^{2} \mathbf{k}_{\perp}}{\mathbf{k}_{\perp}^{2}} P_{q \rightarrow g q}^{\mathrm{med}}\left(x, \mathbf{k}_{\perp}\right),
\end{aligned}
$$

where $P_{i \rightarrow j k}^{\text {med }}$ is the medium induced corrections to splitting functions. The medium correction to the total quark jet function is [5]

$$
J_{q}^{\mathrm{med}}(E, R, \mu)=\frac{\alpha_{s}(\mu)}{2 \pi^{2}} \int_{0}^{1} d x \int_{0}^{2 E x(1-x) \tan R / 2} \frac{d^{2} \mathbf{k}_{\perp}}{\mathbf{k}_{\perp}^{2}} P_{q \rightarrow q g}^{\text {med,real }}\left(x, \mathbf{k}_{\perp}\right) .
$$

The fragmentation functions in medium evolve according to a medium-modified DGLAP evolution. The evolution in medium has been used quite widely in literature $[8,11,12,17]$. In our work, the evolution of the fragmentation function is given by

$$
\frac{d}{d \ln \mu} \tilde{D}_{q}^{Q, \text { full }}(\kappa, \mu)=\frac{\alpha_{s}(\mu)}{\pi}\left(\tilde{P}_{q q}(\kappa)+\tilde{P}_{q q}^{\mathrm{med}}(\kappa, \mu)\right) \tilde{D}_{q}^{Q, \text { full }}(\kappa, \mu) .
$$

The average jet charge in heavy-ion collisions up to first order in QCD and first order in opacity can be written as

$$
\left\langle Q_{q, K}^{\mathrm{AA}}\right\rangle=\left\langle Q_{q, K}^{\mathrm{pp}}\right\rangle\left(1+\tilde{\mathcal{J}}_{q q}^{\mathrm{med}}-J_{q}^{\mathrm{med}}\right) \exp \left[\int_{\mu_{0}}^{\mu} \frac{d \bar{\mu}}{\bar{\mu}} \frac{\alpha_{s}(\bar{\mu})}{\pi} \tilde{P}_{q q}^{\mathrm{med}}\right]+O\left(\alpha_{s}^{2}, \chi^{2}\right) .
$$

If we choose the scale $\mu=p_{T} R$, the $p_{T}$ dependence of the jet charge is

$$
\frac{d}{d \ln p_{T}} \ln \left\langle Q_{q, \kappa}^{\mathrm{AA}}\right\rangle=\frac{\alpha_{s}\left(p_{T} R\right)}{\pi}\left[\tilde{P}_{q q}(\kappa)+\tilde{P}_{q q}^{\mathrm{med}}\left(\kappa, p_{T} R\right)+\int_{0}^{1} d x\left(x^{\kappa}-1\right) P_{q q}^{\mathrm{med}}\left(\kappa, k_{\perp}=x(1-x) p_{T} R\right)\right] .
$$

In principle the scale violation in heavy-ion collisions can be used to extract the information of the medium splitting functions.

\section{Numerical results}

Only one nonperturbative parameter for a given jet flavor is required for jet charge distribution, which is obtained by fitting our predictions using PYTHIA8 $[18,19]$ simulations or ALTAS measurements [4]. In QCD medium we evolve the fragmentation functions from $\Lambda_{\mathrm{QCD}}=0.2 \mathrm{GeV}$ to the jet scale $p_{T} R$. The scale of the QCD coupling in medium is set to be $\sqrt{k_{\perp}^{2}+\mu_{D}^{2}}$ where $\mu_{D}=0.75 \mathrm{GeV}$. The flavor fractions of jet types are simulated by LO matrix elements with CT14NLO PDF sets [20] for proton and nCTEQ15 [21] PDF sets for nuclei. In heavy-ion collisions for the jet fractions, we choose to use the energy loss approach [11].

In Fig. 1, we present the up- and down-quark jet charges in $\mathrm{p}+\mathrm{p}$ collisions with $\sqrt{s_{\mathrm{NN}}}=8 \mathrm{TeV}$ compared with ATLAS measurements [4]. The bands of the predictions are generated by combining the scale uncertainties and $1-\sigma$ deviation of the nonperturbative parameter. We find that these scale uncertainties are small. As indicated in the jet charge definition, the absolute value is smaller for larger 

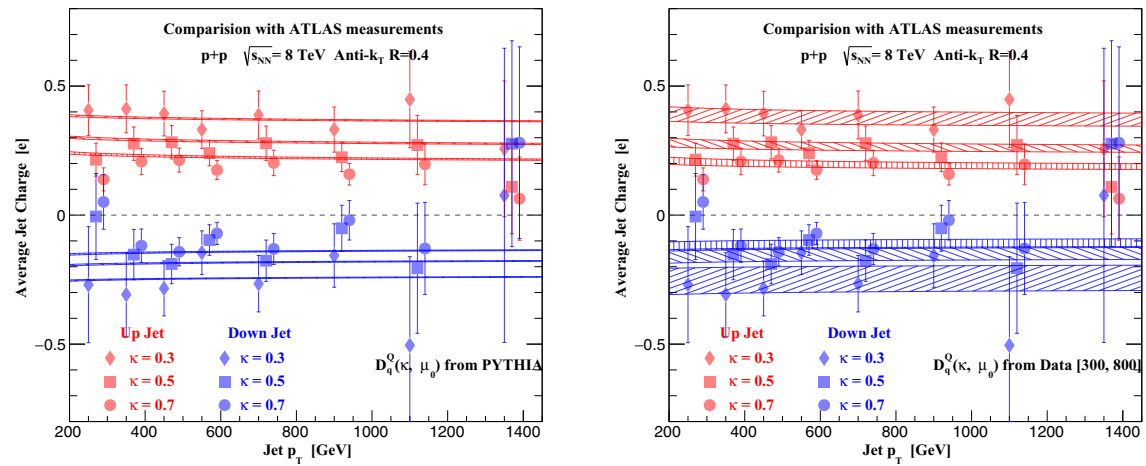

Figure 1. Average charge of up and down-quark jet as a function of jet $p_{T}$ with $\kappa=0.3,0.5$ and 0.7 in $\sqrt{s_{\mathrm{NN}}}=$ $8 \mathrm{TeV} \mathrm{p}+\mathrm{p}$ collisions. The dots with error bars represent the measurements by ATLAS [4]. The red and blue bands are the predictions with the non-perturbative parameters obtained through fitting to PYTHIA simulations (left) and ATLAS measurements (right).
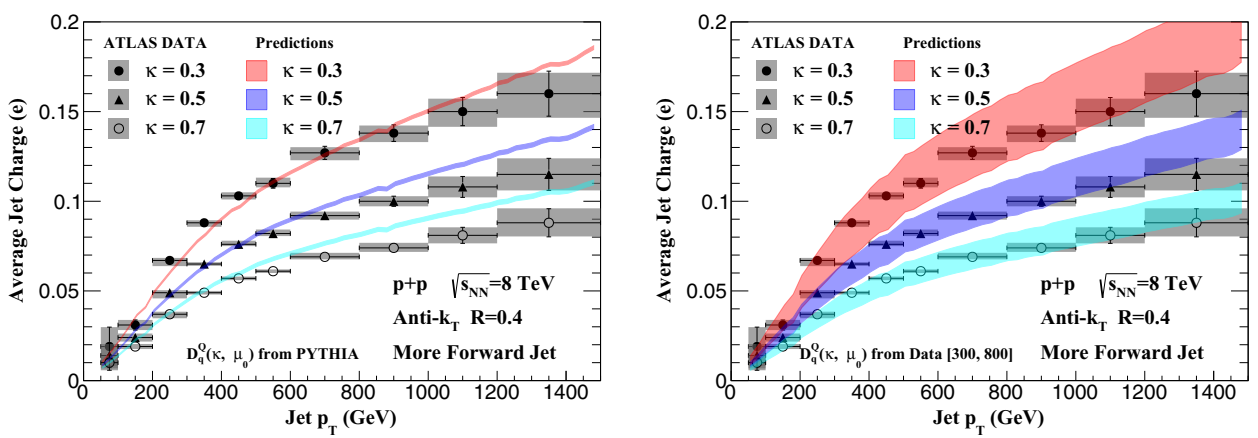

Figure 2. Transverse momentum dependence of the average jet charge distributions for more forward jets in dijet events at $\sqrt{s_{\mathrm{NN}}}=8 \mathrm{TeV}$ in $\mathrm{p}+\mathrm{p}$ collisions. The non-perturbative parameters are obtained by fitting to PYTHIA simulations (left) and ATLAS measurements (right).

$\kappa$. The up-quark and down-quark jets are well separated with positive and negative electric charges, respectively. The predictions are consistent with the measurements by ATLAS.

Considering valence quark contribution only, the charge for more forward jets is $\left\langle Q_{\kappa}^{f}\right\rangle=\left(f_{u}^{f}-\right.$ $\left.f_{\bar{u}}^{f}\right)\left\langle Q_{\kappa}^{u}\right\rangle+\left(f_{d}^{f}-f_{\bar{d}}^{f}\right)\left\langle Q_{k}^{d}\right\rangle$, where the more forward jet is the one with a larger $\eta$ in dijet events. $f_{q}^{f}$ represents the fraction of jet flavors. $Q_{\kappa, q_{i}}$ is the $q_{i}$-jet's charge. For more forward jets, the valence quark contribution is enhanced, especially in the high transverse momentum region. In Fig. 2 we show the average jet charge distribution for the more forward jet. Our predictions can describe the experimental data very well.

In the rest of this section we will use the fragmentation functions obtained from PYTHIA simulations. The left panel in Fig. 3 shows the average jet charge distribution and its modifications with $\sqrt{s_{\mathrm{NN}}}=5.02 \mathrm{TeV}$ in $0-10 \%$ central $\mathrm{Pb}+\mathrm{Pb}$ collisions. The uncertainties are calculated by varying the effective coupling $g \in[1.8,2.0]$ between the energetic parton and QCD medium. The charges of up- and down-quark jets remain well separated. The right panel in Fig. 3 shows the modification of up-quark jet charge. Because the QCD interaction is flavor-blind, the medium modifications for up- 

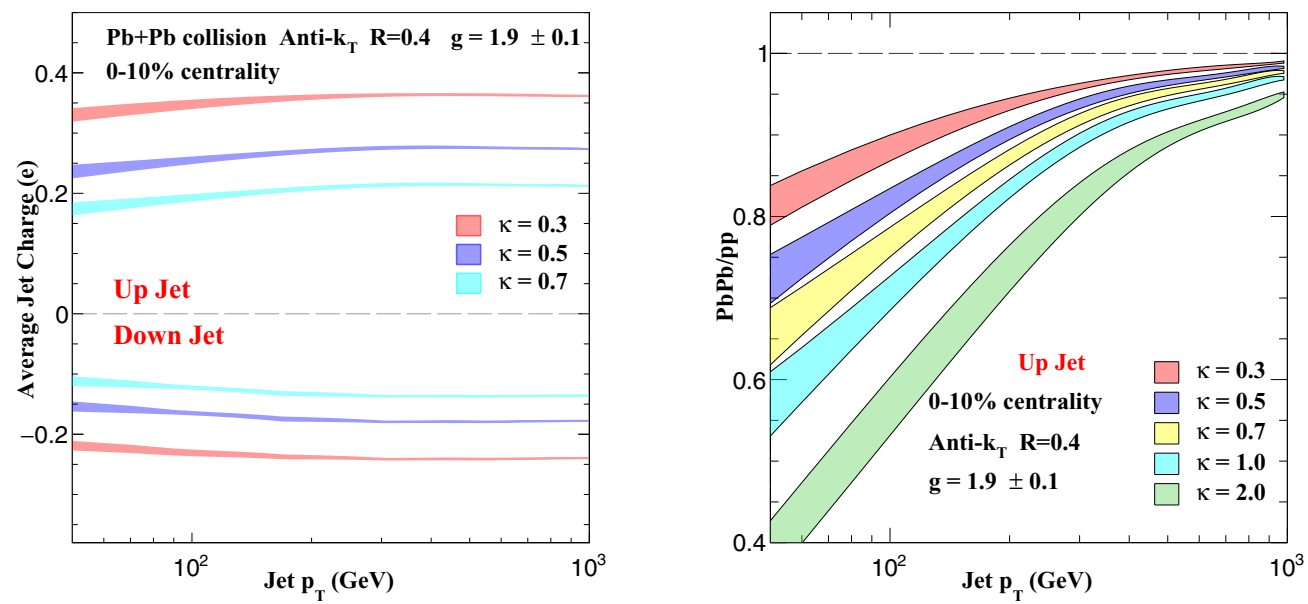

Figure 3. Average quark jet charges and their modifications as a function of jet $p_{T}$ in $\sqrt{s_{\mathrm{NN}}}=5.02 \mathrm{TeV} 0-10 \%$ central $\mathrm{Pb}+\mathrm{Pb}$ collisions. The coupling between the jet and QCD medium is set to be $g=1.9 \pm 0.1$.
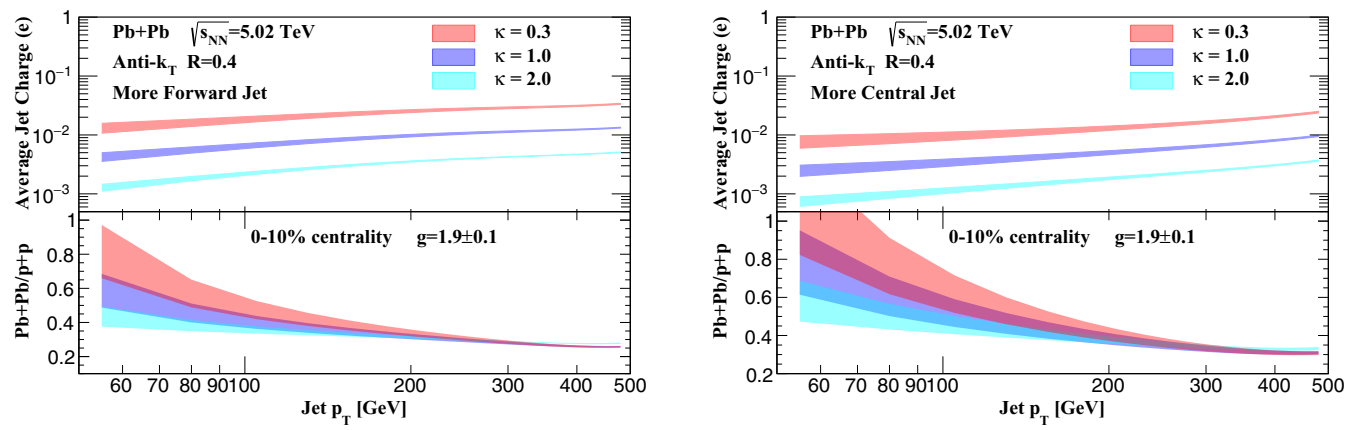

Figure 4. Average jet charge distribution for more forward (right) and more central (left) jet with $\kappa=0.3,1.0$ and 2.0 in $\mathrm{Pb}+\mathrm{Pb}$ collisions with $\sqrt{s_{\mathrm{NN}}}=5.02 \mathrm{TeV}$ with nonperturbative parameters from PYTHIA. The bottom panels show the modification of the average jet charge in $0-10 \%$ central $\mathrm{Pb}+\mathrm{Pb}$ collisions relative to the ones in $\mathrm{p}+\mathrm{p}$ collisions.

and down-quark jet are the same. The modifications are larger for smaller energy jet or larger $\kappa$, which arises from the medium-induced parton shower on jet evolution.

Figure 4 presents our predictions for the average charge of more forward and more central jets in $\mathrm{Pb}+\mathrm{Pb}$ collision with $\sqrt{s_{\mathrm{NN}}}=5.02 \mathrm{TeV}$, which can be measured straightforwardly in heavy-ion collisions. Due to isospin effects, there is a huge cancellation between the contributions from up- and down-quark jets. In results, average charge is smaller in $\mathrm{Pb}+\mathrm{Pb}$ collisions, especially for very large $p_{T}$. At low $p_{T}$ the modification is sensitive to medium induced radiations, which at high $p_{T}$ the initial stat effects are more important.

\section{Conclusion}

In these proceedings, we reported a theoretical framework to investigate average jet charge in heavyion collisions, which is build upon the $\mathrm{SCET}_{\mathrm{G}}$ approach. The jet charge observable can be written 
as the product of the jet matching function and fragmentation functions. In heavy-ion collisions the modifications of the collinear objects are constructed with the help of the medium-induced splitting kernels derived with $\mathrm{SCET}_{\mathrm{G}}$. We presented the average jet charge distributions for specific jet flavor, as well as the predictions for more forward and central jets at hadronic colliders. The jet charge does not depend on the hard process, which can also be measured at RHIC and a future EIC.

\section{References}

[1] R.D. Field, R.P. Feynman, Nucl. Phys. B136, 1 (1978), [763(1977)]

[2] D. Krohn, M.D. Schwartz, T. Lin, W.J. Waalewijn, Phys. Rev. Lett. 110, 212001 (2013), 1209.2421

[3] W.J. Waalewijn, Phys. Rev. D86, 094030 (2012), 1209. 3019

[4] G. Aad et al. (ATLAS), Phys. Rev. D93, 052003 (2016), 1509.05190

[5] H.T. Li, I. Vitev (2019), 1908.06979

[6] G. Ovanesyan, I. Vitev, JHEP 06, 080 (2011), 1103 . 1074

[7] G. Ovanesyan, I. Vitev, Phys. Lett. B706, 371 (2012), 1109.5619

[8] Z.B. Kang, F. Ringer, I. Vitev, JHEP 03, 146 (2017), 1610.02043

[9] M.D. Sievert, I. Vitev, Phys. Rev. D98, 094010 (2018), 1807.03799

[10] M.D. Sievert, I. Vitev, B. Yoon, Phys. Lett. B795, 502 (2019), 1903.06170

[11] Z.B. Kang, R. Lashof-Regas, G. Ovanesyan, P. Saad, I. Vitev, Phys. Rev. Lett. 114, 092002 (2015), 1405.2612

[12] Y.T. Chien, A. Emerman, Z.B. Kang, G. Ovanesyan, I. Vitev, Phys. Rev. D93, 074030 (2016), 1509.02936

[13] Z.B. Kang, F. Ringer, I. Vitev, Phys. Lett. B769, 242 (2017), 1701.05839

[14] H.T. Li, I. Vitev, JHEP 07, 148 (2019), 1811.07905

[15] A. Jain, M. Procura, W.J. Waalewijn, JHEP 05, 035 (2011), 1101 . 4953

[16] S.D. Ellis, C.K. Vermilion, J.R. Walsh, A. Hornig, C. Lee, JHEP 11, 101 (2010), 1001.0014

[17] H.T. Li, I. Vitev, Phys. Lett. B793, 259 (2019), 1801.00008

[18] T. Sjostrand, S. Mrenna, P.Z. Skands, Comput. Phys. Commun. 178, 852 (2008), 0710. 3820

[19] T. Sjöstrand, S. Ask, J.R. Christiansen, R. Corke, N. Desai, P. Ilten, S. Mrenna, S. Prestel, C.O. Rasmussen, P.Z. Skands, Comput. Phys. Commun. 191, 159 (2015), 1410. 3012

[20] S. Dulat, T.J. Hou, J. Gao, M. Guzzi, J. Huston, P. Nadolsky, J. Pumplin, C. Schmidt, D. Stump, C.P. Yuan, Phys. Rev. D93, 033006 (2016), 1506.07443

[21] K. Kovarik et al., Phys. Rev. D93, 085037 (2016), 1509.00792 